
\title{
PREVALENCIA DE SINTOMAS MÚSCULO-ESQUELÉTICOS EM
TRABALHADORAS DE ENFERMAGEM
}

\author{
Giovana Pimentel Gurgueira ${ }^{2}$ \\ Neusa Maria Costa Alexandre ${ }^{3}$ \\ Heleno Rodrigues Corrêa Filho ${ }^{4}$
}

Gurgueira GP, Alexandre NMC, Corrêa HR Filho. Prevalência de sintomas músculo-esqueléticos em trabalhadoras de enfermagem. Rev Latino-am Enfermagem 2003 setembro-outubro; 11(5):608-13.

Este trabalho teve por objetivo avaliar sintomas músculo-esqueléticos em trabalhadoras de enfermagem. Os sujeitos compreenderam 105 auxiliares e técnicos de enfermagem que trabalhavam em unidades de internação de um hospital que atende pacientes com alto grau de dependência física. Utilizou-se um questionário contendo dados demográficos e ocupacionais e queixas osteomusculares, dentro de uma abordagem ergonômica, adaptado do Nordic Musculoskeletal Questionnaire. Das participantes, 93\% referiram algum tipo de sintoma osteomuscular nos últimos 12 meses. As mais elevadas prevalências desses sintomas, segundo as áreas anatômicas, foram: região lombar (59\%), ombros (40\%), joelhos (33,3\%) e região cervical (28,6\%). Verificou-se também que, das respondentes, 29,5\% faltaram ao trabalho e 47,6\% consultaram um médico nos últimos 12 meses devido aos mesmos sintomas. Observou-se também menor tempo de trabalho na área de enfermagem e na unidade atual entre as trabalhadoras com ocorrência maior de dor nos joelhos $(p=0,0272)$ e dor lombar $(p=0,0332)$, respectivamente. No entanto, a dor em punhos/mãos mostrou-se mais freqüente entre as participantes com maior número de horas semanais trabalhadas $(O R=3,72<O R<11,12 ; p=0,0141)$.

DESCRITORES: ergonomia; dor lombar; enfermagem

\section{SELF-REPORTED MUSCULOSKELETAL SYMPTOMS AMONG NURSING PERSONNEL}

The aim of this cross-sectional study was to evaluate musculoskeletal symptoms among female members of the nursing personnel. The sample consisted of 105 female nursing aides and technicians who were working at a university hospital with highly dependent patients. The questionnaire was composed of items on demographic and job-factor information and the symptom section was a modification of the "Nordic Musculoskeletal Questionnaire". In this study, 93\% of the health workers reported at least one musculoskeletal symptom in the previous 12 months. The highest prevalence of musculoskeletal disorders was in the following anatomical areas: low back (59\%), shoulders (40\%), knees (33.3\%) and neck (28.6\%). As a result of musculoskeletal pain, 29.5\% of the respondents reported missing work and $47.6 \%$ reported having had an appointment to see a physician in the previous 12 months. Limited experience on the job and in the present unit were also observed among those who had more frequent complaints of pain in the knees $(p=0.0272)$ and low back pain $(p=0.0332)$, respectively. However, hand/ wrist pain occurred more often among the participants with the higher numbers of weekly worked hours $(O R=3.72: 1.26<O R<11.12$; $p=0.0141)$.

DESCRIPTORS: ergonomics; low back pain; nursing

\section{PREVALENCIA DE SÍNTOMAS MÚSCULO-ESQUELÉTICOS EN TRABAJ ADORAS DE ENFERMERÍA}

Este trabajo tuvo por objetivo evaluar síntomas músculo-esqueléticos en trabajadoras de enfermería. Los sujetos fueron 105 auxiliares y técnicas de enfermería que trabajaban en unidades de hospitalización de una institución hospitalaria de pacientes con alto grado de dependencia física. Se utilizó un cuestionario conteniendo datos demográficos y ocupacionales y quejas osteomusculares dentro de un abordaje ergonómico, adaptado del "Nordic Musculoskeletal Questionnaire". De las participantes, 93\% refirieron algún tipo de síntoma osteomuscular en los últimos 12 meses. Las más elevadas prevalencias de esos síntomas según las áreas anatómicas fueron: región lumbar (59\%), hombros (40\%), rodillas (33,3\%) y región cervical (28,6\%). Se verificó también que de las entrevistadas, 29,5\% faltó al trabajo y 47,6\% consultó un médico en los últimos 12 meses debido a los mismos síntomas. Se observó también menor tiempo de trabajo en el área de enfermería y en la unidad actual entre las trabajadoras con mayor ocurrencia de dolor en las rodillas $(p=0,0272)$ y dolor lumbar $(p=0,0332)$, respectivamente. No obstante, el dolor en puños/manos se mostró más frecuente entre las participantes con mayor número de horas semanales trabajadas $(O R=3,72<O R<11,12 ; p=0,0141)$.

DESCRIPTORES: ergonomía; dolor de la región lumbar; enfermería

\footnotetext{
${ }^{1}$ Pesquisa realizada com auxílio de Bolsa de iniciação científica da Fundação de Amparo à Pesquisa do Estado de São Paulo (FAPESP), processo número 01/00783-8; ${ }^{2}$ Enfermeira, Mestranda em Enfermagem; ${ }^{3}$ Enfermeira, Professor Associado, e-mail: neusalex@fcm.unicamp.br; ${ }^{4}$ Epidemiologista, Professor Assistente Doutor. Faculdade de Ciências Médicas da Universidade Estadual de Campinas
} 
INTRODUÇÃO

O trabalho de Enfermagem, desenvolvido essencialmente por mulheres, envolve numerosos fatores de risco para a saúde. As auxiliares são duplamente penalizadas pelo acúmulo de trabalho doméstico, o que contribui para suas piores condições de saúde ${ }^{(1)}$. Os hospitais estão associados à prestação de serviços à saúde, visando a assistência, o tratamento e a cura daqueles acometidos pela doença. No entanto, também são responsáveis pela ocorrência de uma série de riscos à saúde daqueles que ali trabalham. A maioria dos hospitais possui estrutura de alto nível de complexidade e diversidade de serviços e, conseqüentemente, de riscos ocupacionais. Há extensa lista de danos a todos os sistemas orgânicos, entre eles os problemas músculoesqueléticos, que são muito freqüentes na equipe de enfermagem. Pesquisadores e organizações de várias partes do mundo têm destacado a equipe de enfermagem como grupo de risco em relação ao desenvolvimento de distúrbios osteomusculares ${ }^{(2)}$. Entre esses distúrbios, destacam-se as lombalgias. A dor lombar tem sido particularmente bem estudada entre os trabalhadores da saúde, sendo resultado de traumas cumulativos ${ }^{(3-4)}$. As lesões dorsais ocupacionais ocorrem mais freqüentemente quando é realizado o cuidado direto ao paciente, especialmente o levantamento desses ${ }^{(5-8)}$. Muitos desses profissionais retornam ao serviço, apesar de continuarem apresentando os mesmos sintomas ${ }^{(8)}$.

A literatura científica aborda particularmente questões relacionadas às dores lombares, sendo que não se tem conhecimento de estudos nacionais abrangendo especificamente os sintomas músculo-esqueléticos nas diferentes regiões corporais entre os trabalhadores de enfermagem. Dentro desse contexto, o presente estudo teve por objetivos investigar se trabalhadoras de enfermagem apresentaram outros sintomas músculoesqueléticos além da dor lombar e identificar determinados fatores que podem contribuir para o desenvolvimento desses sintomas.

\section{METODOLOGIA}

Descrição dos sujeitos

No presente estudo foram incluídos 105 auxiliares e técnicos de enfermagem do sexo feminino que trabalhavam em unidades de internação que atendem pacientes com alto grau de dependência física, de um hospital universitário. As unidades selecionadas foram: Unidade de Terapia Intensiva, Cirurgia do Trauma, Emergência Clínica, Ortopedia, Traumatologia, Neuroclínica e Neurocirurgia. Foram excluídas as funcionárias que estavam em licença saúde ou outro tipo de afastamento durante o período da coleta de dados. A participação neste estudo foi voluntária e todos os sujeitos que concordaram em participar assinaram termo de consentimento.

Instrumentos de coleta de dados

O estudo foi norteado por um questionário composto por duas partes: a primeira continha questões sobre determinadas características demográficas e ocupacionais, e a segunda avaliava as queixas músculoesqueléticas.

Dados demográficos e ocupacionais: contém questões sobre idade, estado civil, categoria profissional, turno e unidade de trabalho, tempo que trabalha em enfermagem e na unidade, horas trabalhadas por semana, e atividades ocupacionais consideradas como causas de lombalgia. Essa parte do questionário foi desenvolvida tendo como suporte teórico outra investigação ${ }^{(3)}$. Essa parte do instrumento foi submetida à apreciação de três especialistas do Departamento de Enfermagem da Faculdade de Ciências Médicas (FCM) da Universidade Estadual de Campinas (UNICAMP) para a avaliação de sua objetividade, adequação e conteúdo. Realizou-se também um teste piloto com 20 trabalhadoras de enfermagem que não estavam envolvidas no projeto. Posteriormente, foram realizadas algumas modificações, obtendo-se a versão final do instrumento.

Sintomas músculo-esqueléticos: para avaliar as queixas osteomusculares, utilizou-se um instrumento derivado do Standardized Nordic Questionnaire ${ }^{(9)}$. Esse instrumento é utilizado internacionalmente e foi desenvolvido para padronizar pesquisas sobre investigações músculo-esqueléticas. Tem como um dos objetivos principais avaliar distúrbios osteomusculares dentro de uma abordagem ergonômica. O questionário contém uma figura humana vista pela região posterior, dividida em nove regiões anatômicas: região cervical, ombros, região torácica, cotovelos, punhos/mãos, região lombar, quadril/coxas, joelhos, tornozelos/pés. As questões estão relacionadas com cada área anatômica e verificam se os respondentes tiveram dores nos últimos 12 meses e sete dias; procuram também investigar se essas pessoas precisaram faltar ao serviço ou procurar auxílio médico nos últimos 12 meses devido aos mesmos sintomas. A escolha desse questionário deve-se ao fato 
de já ter sido validado na cultura brasileira, e também por ser considerado instrumento simples, com questões diretas e de fácil compreensão ${ }^{(10)}$.

Coleta e análise dos dados

Os dados foram coletados por meio de entrevista individual por um dos autores no período de 01 de abril a 31 de maio de 2001. As participantes foram entrevistadas nos seus respectivos locais de trabalho. Os dados foram inseridos no programa Microsoft Excel, versão 2000. A análise foi realizada com o auxílio do Setor de Estatística da FCM da UNICAMP. Realizou-se análise descritiva das variáveis. Verificou-se também se existia associação entre as variáveis: idade, estado civil, categoria, turno e unidade de trabalho, tempo que trabalha em enfermagem e na unidade e horas trabalhadas por semana com a presença de sintomas músculo-esqueléticos nos últimos 12 meses. Para isso, compararam-se os grupos com dor e sem dor, nas diferentes regiões corporais, utilizando o teste Quiquadrado. Quando os valores esperados foram menores que 5, utilizou-se o teste exato de Fisher, sendo considerada significativa a associação quando o $\mathrm{p}$-valor $\leq$ 0,05. A análise estatística foi executada com auxílio do programa The SAS System for Windows, versão 6.12, 1996.

Aspectos éticos

O protocolo (número 352/2000) obteve o parecer favorável do Comitê de Ética da Instituição. As trabalhadoras foram convidadas a participar do estudo, quando receberam informações sobre os objetivos do mesmo e esclarecimentos de dúvidas. As funcionárias que concordaram em participar do estudo assinaram termo de consentimento.

\section{RESULTADOS}

Dados gerais e demográficos

Entrevistou-se o total de 105 trabalhadoras de enfermagem, representando taxa de participação de $95,5 \%$. Dessas, $66 \%$ eram auxiliares de enfermagem e $34 \%$ eram técnicas de enfermagem. A idade média das participantes era de 36,5 anos (D.P. $=9$ e idades entre 19 e 58 anos). Cerca de $50 \%$ das entrevistadas possuem companheiro. O tempo de trabalho em enfermagem foi em média 10,7 anos, sendo que especificamente no setor atual foi de 5,8 anos. Em relação às horas trabalhadas por semana, verificou-se que $54,3 \%$ das respondentes relataram trabalhar mais de 40 horas semanais. Como o hospital estudado exige carga de trabalho semanal de 32 horas, pode-se inferir que as funcionárias possuem outro emprego ou continuação da jornada de trabalho através de hora extra. Em relação à unidade de trabalho, 29,5\% das entrevistadas pertenciam à Unidade de Terapia Intensiva, 27,6\% à Emergência Clínica e Cirurgia do Trauma, 14,3\% à Ortopedia e Traumatologia, e 28,6\% à Neuroclínica e Neurocirurgia. Quanto ao turno de trabalho, $32,4 \%$ das entrevistadas trabalhavam no período da manhã, $26,7 \%$ à tarde e $40,9 \%$ à noite.

Sintomas músculo-esqueléticos

Independentemente da região afetada, 93\% das participantes referiram algum tipo de sintomas osteomusculares nos últimos 12 meses, e $62 \%$ nos últimos sete dias.

A Figura 1 mostra a prevalência de sintomas músculo-esqueléticos anual e semanal.

\begin{tabular}{|l|c|c|}
\hline \multicolumn{1}{|c|}{ Região } & $\begin{array}{c}\text { Presença de dor } \\
\text { nos últimos 12 } \\
\text { meses em (\%) }\end{array}$ & $\begin{array}{c}\text { Presença de dor } \\
\text { nos últimos 7 } \\
\text { dias em (\%) }\end{array}$ \\
\hline Cervical & 28,6 & 14,3 \\
\hline Ombros & 40,0 & 16,2 \\
\hline Torácica & 21,9 & 10,5 \\
\hline Cotovelos & 07,6 & 5,7 \\
\hline Punhos/mãos & 24,8 & 14,3 \\
\hline Lombar & 59,0 & 31,4 \\
\hline Quadril/coxas & 16,2 & 5,7 \\
\hline Joelhos & 33,3 & 14,3 \\
\hline Tornozelos/pés & 14,3 & 7,6 \\
\hline
\end{tabular}

Figura 1 - Porcentagem de trabalhadoras de enfermagem referindo sintomas músculo-esqueléticos nas diferentes regiões corporais. Campinas, 2001 
As participantes apresentaram as mais elevadas taxas de distúrbios osteomusculares nos últimos 12 meses nas seguintes regiões anatômicas: lombar (59\%), ombros (40\%), joelhos (33,3\%) e região cervical $(28,6 \%)$. Em relação à presença de dor nos últimos 7 dias, a região lombar continuou sendo a mais citada $(31,4 \%)$, seguida pelos ombros (16,2\%). É importante destacar a elevada presença de dor em áreas não relacionadas com a coluna vertebral em um período de 12 meses, tais como ombros (40\%), joelhos (33,3\%) e punhos/mãos $(24,8 \%)$.

Para avaliar indicadores de gravidade dos sintomas, pesquisou-se o absenteísmo e a procura por auxílio médico, que podem ser analisados na Figura 2.

\begin{tabular}{|l|c|c|}
\hline \multicolumn{1}{|c|}{ Região } & $\begin{array}{c}\text { Ausência do } \\
\text { trabalho devido a } \\
\text { essa condição (\%) }\end{array}$ & $\begin{array}{c}\text { Consulta médica } \\
\text { nos últimos 12 } \\
\text { meses devido a } \\
\text { essa condição (\%) }\end{array}$ \\
\hline Cervical & 5,7 & 9,5 \\
\hline Ombros & 3,8 & 11,4 \\
\hline Torácica & 2,9 & 4,8 \\
\hline Cotovelos & 1,0 & 2,9 \\
\hline Punhos/mãos & 7,6 & 2,7 \\
\hline Lombar & 13,3 & 9,5 \\
\hline Quadril/coxas & 5,7 & 13,3 \\
\hline Joelhos & 5,7 & 3,8 \\
\hline Tornozelos/pés & 2,9 & \\
\hline
\end{tabular}

Figura 2 - Porcentagem de trabalhadoras de enfermagem referindo ausência no trabalho e procura por auxílio médico devido a sintomas músculo-esqueléticos, nos últimos 12 meses. Campinas, 2001

A dor lombar foi a queixa mais freqüente para justificar a ausência no trabalho e procura de auxílio médico. Dos respondentes, $13,3 \%$ referiram ter se ausentado do trabalho devido a dor lombar, e $25,7 \%$ visitaram um médico pelo mesmo problema em um período de 12 meses. Observou-se também que $24,7 \%$ das participantes referiram ter procurado ajuda médica devido a dor nos joelhos e ombros. De uma forma geral, $29,5 \%$ faltaram ao serviço e $47,6 \%$ consultaram um médico tendo como justificativa os problemas osteomusculares.

Atividades ocupacionais e fatores associados

As participantes também responderam quais as atividades ocupacionais que elas achavam que causavam dor lombar. Os procedimentos mais citados estavam relacionados com a movimentação e transporte de pacientes. As trabalhadoras relataram, em primeiro lugar, movimentar pacientes $(87,6 \%)$ e, em seguida, o transporte dos mesmos (49,5\%).

Em relação aos fatores de risco, não foi encontrada associação estatisticamente significante entre as variáveis: idade, estado civil, categoria profissional, turno e unidade de trabalho com os sintomas osteomusculares nos últimos 12 meses nas diferentes regiões anatômicas. Observou-se menor tempo de trabalho em serviço de enfermagem e na unidade atual de trabalho entre as trabalhadoras com ocorrência maior de dor nos joelhos ( $p=0,0272)$ e dor lombar ( $p=0,0332)$, respectivamente. $A$ relação inversa à esperada entre tempo de exposição e sintomas é certamente decorrente de viés de sobrevivência típico do desenho transversal. Essa hipótese fica reforçada diante do grau de incapacidade que implica a dor nessas regiões corporais, com repercussão sobre a permanência na atividade laboral. A mesma limitação incapacitante não se verifica com a dor em punho/mãos, que se mostrou mais freqüente entre as pessoas com maior número de horas semanais trabalhadas $(\mathrm{OR}=3,72 ; 1,26<\mathrm{OR}<11,12$; $\mathrm{p}=0,0141)$.

\section{DISCUSSÃO}

No presente estudo, procurou-se ampliar os conhecimentos sobre as queixas músculo-esqueléticas na equipe de enfermagem brasileira. Salienta-se que o estudo foi realizado somente com auxiliares e técnicas de enfermagem que trabalhavam em enfermarias em que havia pacientes com alto grau de dependência física. Essas duas categorias profissionais executam os mesmos tipos de atividades ocupacionais.

Os resultados demonstraram que as trabalhadoras de enfermagem relataram elevada ocorrência de sintomas músculo-esqueléticos em diversas regiões do corpo num 
período de 12 meses (93\%) e de sete dias (62\%). Estudos internacionais confirmam esses dados, revelando a importância desse problema entre profissionais da enfermagem $^{(11-13)}$. Em um estudo ${ }^{(14)}$ realizado com a equipe de enfermagem prestando cuidados domiciliares, na Holanda, encontrou-se prevalência de $63 \%$ de queixas osteomusculares, ao passo que em outro estudo ${ }^{(2)}$ foi obtida prevalência anual de $84 \%$ na equipe de enfermagem do sexo feminino de um hospital, na Suécia. Em relação às regiões mais citadas como responsáveis por sintomas músculo-esqueléticos nos últimos 12 meses, a lombar ficou em primeiro lugar, seguida pelos ombros, joelhos e região cervical. Estudo ${ }^{(2)}$, realizado em funcionárias de enfermagem de um hospital, da Suécia, encontrou achados similares, mostrando sintomas principalmente na região lombar (65\%), seguida pelos ombros (60\%) e região cervical (53\%). Pesquisa similar ${ }^{(11)}$ verificou a prevalência de sintomas na região lombar (56\%), nos ombros (53\%), na região cervical (48\%) e nos joelhos $(30 \%)$. Outro estudo ${ }^{(12)}$, realizado em pessoas cuidadoras de idosos, na Holanda, observou a presença de sintomas na região lombar (34\%), na região cervical (23\%) e nos ombros (19,5\%). Os dados encontrados no presente estudo demonstram que os trabalhadores da área de saúde brasileiros apresentam taxas similares de prevalência de distúrbios osteomusculares comparados com países desenvolvidos. As regiões corporais mais atingidas parecem ser a lombar, ombros, cervical e joelhos. Os instrumentos utilizados não permitiram avaliar a intensidade dos sintomas.

Em relação à avaliação da capacidade funcional, a dor lombar demonstrou ser uma das maiores causas de absenteísmo e de procura por ajuda médica entre os participantes do presente estudo. Foram também observadas taxas elevadas de procura por auxílio médico em um estudo holandês, realizado na equipe de enfermagem que assiste pacientes incapacitados e geriátricos. Estes autores ${ }^{(12)}$ encontraram que, das pessoas estudadas, $61 \%$ procuraram tratamento devido à presença de dor em braço/região cervical, 51\% nas costas e $47 \%$ nas pernas. A questão da influência das lombalgias em produzir incapacidade ao trabalho e absenteísmo tem sido bem documentada pela literatura científica $^{(15)}$. No entanto, novas pesquisas precisam abordar a severidade dos sintomas nas outras regiões corporais.

Pesquisas internacionais têm mostrado que as afecções músculo-esqueléticas são causadas por inúmeros fatores individuais, físicos e psicossociais. Dentre esses, os fatores ocupacionais têm sido muito estudados, sendo que apresentam valores diferentes de acordo com a área corporal envolvida. No presente estudo, verificouse que as participantes acreditam que a dor lombar é causada principalmente por procedimentos relacionados com a movimentação e transferência de pacientes. Estudos já têm comprovado a importante participação da manipulação de pacientes em lesões na região dorsal em trabalhadores de enfermagem ${ }^{(7-8)}$. Como exemplo dessas atividades, foram citadas nas entrevistas: ajustar 0 paciente na cama, transferir o paciente da cama para a maca/cadeira de rodas e vice-versa, dar banho de leito. Essas atividades também foram citadas por outros autores, que as complementam, dizendo que aparentemente nenhuma das técnicas atuais de transferência oferece proteção suficiente à equipe de enfermagem, e que isso pode explicar a alta prevalência de problemas dorsais entre esses trabalhadores ${ }^{(6)}$.

Outros estudos têm procurado avaliar as possíveis causas de problemas osteomusculares em trabalhadores da área da saúde, levando em consideração as diferentes regiões corporais envolvidas ${ }^{(11,16)}$. Os resultados ainda geram controvérsias, mas apóiam a idéia de que os fatores ocupacionais na enfermagem são diferentes, dependendo da região corporal afetada. Estudo ${ }^{(13)}$ realizado com enfermeiras de um hospital encontrou que a dor na região lombar foi devido à movimentação de pacientes e manutenção de posição em flexão. As queixas nos ombros e região cervical foram causadas pela manutenção de posturas estáticas por tempo prolongado ao prestar assistência aos pacientes. Já arrastar ou empurrar camas ocasionaram dores nos ombros e braços.

Em relação aos fatores de risco tempo de trabalho na enfermagem e na unidade atual, obteve-se dados que merecem estudos mais amplos. Pode-se supor algumas explicações para o fato de funcionárias com menor tempo de enfermagem e na unidade estarem apresentando ocorrência maior de dor nos joelhos e região lombar. Um dos mecanismos explicativos seria a questão da existência de uma fase de adaptação. Outro seria o afastamento ou mudança de local de trabalho daquelas com sintomas persistentes. Não se pode esquecer que $93 \%$ das trabalhadoras apresentaram sintoma em alguma região do corpo e isso pode também estar interferindo nos resultados parciais. Outro fato singular foi a associação entre carga horária prolongada com a queixa de dor em 
punhos/mãos. O risco de dor nessa região é válido e também, provavelmente, subestimado, tendo em vista que trabalhadoras com dor incapacitante, caso estivessem presentes neste estudo, aumentariam o valor do Odds Ratio. Os dados encontrados justificam a necessidade de novas pesquisas considerando as diferentes áreas corporais. Também não se pode esquecer a importância dos fatores psicossociais ocupacionais relacionados com a presença de distúrbios osteomusculares na área de saúde, que não foram considerados na pesquisa ${ }^{(17)}$.

Novos estudos precisam ser executados com outras categorias profissionais de enfermagem e em outros locais de trabalho. A comparação dos resultados com outras pesquisas são de difícil execução, visto que utilizam diferentes instrumentos, levam em consideração períodos diferentes para avaliar a prevalência, e estudam inúmeras categorias profissionais que diferem entre os países.

\section{REFERÊNCIAS BIBLIOGRÁFICAS}

1. Silva VEF, Massarollo MCKB. A qualidade de vida e a saúde do trabalhador de enfermagem. Mundo Saúde 1998; 22(5):283-6.

2. Josephson M, Lagerstrom M, Hagberg M, Hjelm EW. Musculoskeletal symptoms and job strain among nursing personnel: a study over a three year period. Occup Environ Med 1997; 54:681-5.

3. Alexandre NMC, Angerami ELS, Moreira DC Filho. Dores nas costas e enfermagem. Rev Esc Enfermagem USP 1996; 30(2):267-84.

4. Smedley J, Inskip H, Cooper C, Coogon D. Natural history of low back pain: a longitudinal study in nurses. Spine 1998; 23(22):2422-6.

5. Alexandre NMC, Benatti MCC. Acidentes de trabalho afetando a coluna vertebral: um estudo realizado com trabalhadores de enfermagem de um hospital universitário. Rev Latino-am Enfermagem 1998; 6(2):65-72.

6. Marras WS. Occupational low back disorder causation and control. Ergonomics 2000; 43(7):880-902.

7. Goldman RH, Jarrard MR, Kim R, Loomis S, Atkins EH. Prioritizing back injury risk in hospital employees: application and comparison of different injury rates. JOM 2000; 42(6):64552.

8. Retsas A, Pinikahana J. Manual handling activities and injuries among nurses: an Australian hospital study. J Adv Nurs 2000; 31(4):875-83.

9. Kuorinka I, Jonsson B, Kilbom A, Vinterberg H, BieringSorensen F, Andersson G, et al. Standardized nordic questionnaires for the analysis of musculoskeletal symptoms. Appl Ergon 1987; 18(3):233-7.

10. Barros ENC, Alexandre NMC. Cros-cultural adaptation of the Nordic musculoskeletal questionnaire. Int Nurs Rev 2003; 50(2):101-8.
Outros estudos precisam ser realizados avaliando a intensidade dos sintomas e considerando os aspectos organizacionais do processo de trabalho na área da saúde.

\section{CONCLUSÕES}

Os resultados demonstram que é elevada a ocorrência de sintomas músculo-esqueléticos em múltiplas regiões corporais, atingindo principalmente a região lombar, ombros, joelhos e região cervical. A dor lombar continua sendo o fator principal de absenteísmo e procura por auxílio médico. Os procedimentos relacionados com a movimentação e transporte de pacientes são considerados os principais causadores de dor na região lombar, indicando que as atividades de cuidado direto aos pacientes podem ser fator de risco para a equipe de enfermagem.

11. Lagerstrom $M$, Wenemark $M$, Hagberg $M$, Hjelm EW. Occupational and individual factors related to musculoskeletal symptoms in five body regions among Swedish nursing personnel. Int Arch Occup Environ Health 1995; 68:27-35.

12. Engels JA, Van Der Gulden JW, Senden TF, Van't Hof, B. Work related risk factors for musculoskeletal complaints in the nursing profession: results of a questionnaire survey. Occup Environ Med 1996; 53:636-41.

13. Botha WE, Bridger RS. Anthropometric variability, equipment usability and musculoskeletal pain in a group of nurses in the Western Cape. Appl Ergon 1998; 29(6):481-90. 14. Engels JA, Van Der Gulden JWJ, Senden TF, Hertog CAWM, Kolk JJ, Binkhorst RA. Physical work load and its assessment among the nursing staff in nursing homes. J Occup Med 1994; 36(3):338-45.

15. Lagerstrom M, Hansson T, Hagberg M. Work-related lowback problems in nursing. Scand J Work Environ Health 1998; 24(6):449-64.

16. Johansson JA. Psychosocial work factors, physical work load and associated musculoskeletal symptoms among home care workers. Scand J Psychol 1995; 36:113-9.

17. Brulin C, Winkvist A. Stress from working conditions among home care personnel with musculoskeletal symptoms. J Adv Nurs 2000; 31(1):181-9. 